\title{
Polymeric porous framework of a bismuth citrate-based complex: A potential vehicle for drug delivery
}

Yang et al., at the Department of Chemistry, University of Hong Kong and Sun Yat-sen University have characterized a series of bismuth citrate complexes by X-ray crystallography and modeled the structure of ranitidine bismuth citrate, a medicine used widely for the treatment of peptic ulcer and gastric reflux disease. The polymeric framework of bismuth citrate may serve as a "drug carrier" for delivery of other drugs in the human body. This significant contribution was reported in SCIENCE CHINA Chemistry 2010, 53(10).

Elements that possess anti-bacterial activity have significant medical applications. Bismuth, the heaviest stable element in the periodic table, exhibits anti-bacterial activity against Helicobacter pylori with minimal toxicity. It is used widely to treat peptic ulcers and gastrointestinal diseases. Ranitidine bismuth citrate (RBC) is a bismuth citrate-based (Bi(cit)-based) drug, developed in 1991. It exhibits efficacy in first-, second- and third-line H. pylori infection therapies when used in combination with other antibiotics, and is more effective than proton pump inhibitor-based (PPI-based) therapy. Because of the importance of Bi(cit)-based drugs, enormous efforts have been made to elucidate their structures. However, there are few structures that reflect the nature of Bi(cit)-based drugs in the stomach.

In this work, the frameworks of four $\mathrm{Bi}$ (cit)-based complexes, obtained under acidic conditions to mimic conditions in the stomach, were resolved. A structural model of RBC was proposed based on the crystal structure of one of these frameworks. The decomposition behavior of $\mathrm{Bi}$ (cit)-based complexes in aqueous solution was investigated using ESI-MS.

$\mathrm{Bi}$ (cit)-based complexes form polymeric porous frameworks with bismuth citrate dimeric units $\left(\left[\mathrm{Bi}(\mathrm{cit})_{2} \mathrm{Bi}\right]^{2-}\right)$ forming the skeleton. Since citrate anions in the frameworks are present in the fully deprotonated, tetraanionic form $\left(\left[\mathrm{C}_{6} \mathrm{H}_{4} \mathrm{O}_{7}\right]^{4-}\right)$, and coordinate with $\mathrm{Bi}^{3+}$ cations, the excess negative charges in these polyanionic frameworks must be balanced by further cations such as $\mathrm{K}^{+}$, $\left[\mathrm{NH}_{4}\right]^{+}$, $\left[\mathrm{H}_{3} \mathrm{NCH}_{2} \mathrm{CH}_{2} \mathrm{NH}_{3}\right]^{2+}$ or $\left[\mathrm{C}_{5} \mathrm{H}_{6} \mathrm{~N}\right]^{+}$. This allows Bi(cit)-based complexes to encapsulate low-molecular mass molecules or ions, either by a diffusion process or via electrostatic interactions, similar to the "uptake process" of cations and small molecules by zeolites. Ranitidine cations were modeled into a simulated structure based on one experimental framework, revealing hydrogen-bond formation between ranitidine molecules and the citrate tetraanions. ESI-MS data indicated that the Bi(cit)-based frameworks degrade from high molecular weight polymers to low molecular weight species in acidic solution.

This paper reports a series of structures of Bi(cit)-based complexes in acidic solution (mimicking the acidic environment of the stomach), establishes an in silico model of a widely used anti-ulcer drug (ranitidine bismuth citrate), and uses experimental ESI-MS data to propose a possible metabolic fate for $\mathrm{Bi}$ (cit)-based drugs under the acidic conditions found in the stomach.

See the article: Yang N, Mao Z W, Sun H Z, et al. Polymeric architectures of bismuth citrate based on dimeric building blocks. Sci China Chem, 2010, 53: 2152-2158, doi: 10.1007/s11426-010-4113-x 